\title{
Robotic Assisted Flexible Ureteroscopy in Covid-19 Positive Patient Using Thulium Fiber Laser: Case Report and Literature Review
}

\author{
Abdulla Al-Ansari ${ }^{1,6}$, Maged Alrayashi' ${ }^{2}$, Hatem Kamkoum ${ }^{2}$, Hossameldin Alnawsara' ${ }^{2}$, Bela Tallai ${ }^{2}$, Tawiz Gul' ${ }^{2}$, Shukri Alfalahi' ${ }^{2}$, Mohamed Abdelka- \\ reem $^{2}$, Mohamed Ali Ebrahim ${ }^{2}$, Fathi Al Kadhi ${ }^{3,6}$, Farhat Ali ${ }^{3}$, Ahmed Ismail ${ }^{3}$, Ibrahim Alnadhari ${ }^{4,5}$, Omar Aboumarzouk ${ }^{1,2,5}$ and Morshed Ali Salah ${ }^{2,5,6^{*}}$ \\ 'Department of Urology, Hamad General Hospital, Hamad Medical Corporation, Qatar \\ ${ }^{2}$ Department of Surgery, Hazm Mebaireek General Hospital, Hamad Medical Corporation, Qatar \\ ${ }^{3}$ Department of Anesthesia, Hazm Mebaireek General Hospital, Hamad Medical Corporation, Qatar \\ ${ }^{4}$ Department of Surgery, Al Wakra Hospital, Hamad Medical Corporation, Qatar \\ ${ }^{5}$ Department of Surgery, Qatar University, Qatar \\ ${ }^{6}$ Department of Surgery, Weill Cornell Medicine, Qatar
}

\begin{abstract}
Flexible Ureteroscopy (FURS) has become an integral aspect of the surgical armamentarium to treat intra-renal stones that are less than $2 \mathrm{~cm}$ in diameter. Despite the progress made with regards to the design of the flexible ureteroscopes, surgeons still need to work with suboptimal ergonomics, which may result in orthopedic complaints, which as a result lead to imperfect performance. Robotic- Assisted FURS with Avicenna Roboflex has provided significant improvement of ergonomics. The Super Pulse Thulium Fiber Laser (SPTFL) may be considered as a viable alternative to holmium laser in stone management. Coronavirus Disease- 19 (Covid-19) has been declared as a pandemic by the World Health Organization (WHO) on March 11, 2020. As a result, many hospitals have been converted to dedicated facilities to manage the Covid-19 patients. Urinary stone disease represents a benign condition, but in non-negligible number of cases, it can lead to potential severe septic complications that could increase the burden on emergency services. Many surgical specialties including urology has made short pathways for patient flow to decrease the contact with the patients which in turn will decrease the possibility of transmission of Covid-19. The use of new technologies such as Avicenna Roboflex and thulium fiber laser in Covid-19 positive patients when performing flexible ureteroscopy can minimize direct contact with the patient, expedite the procedure, while protecting the staff from getting Covid-19 infection.
\end{abstract}

Keywords: Flexible ureteroscopy; Roboflex; Robotic-assisted flexible ureterorenoscopy; Thulium fiber laser; Covid-19

\section{Introduction}

Flexible ureteroscopy has become an integral aspect of the surgical armamentarium to treat intra-renal stones that are less than $2 \mathrm{~cm}$ in diameter [1,2]. Robotic- assisted FURS using Avicenna Roboflex (ELMED, Ankara, Turkey) provides a suitable, safe, and robust platform with significant ergonomic improvement [3-5]. Similarly, the Super Pulse Thulium Fiber Laser (SPTFL) is a new laser technology which is efficient in stone ablation allowing for carrying out stone ablation at a much faster rate, leaving smaller stone fragments [6].

Citation: Al-Ansari A, Alrayashi M, Kamkoum H, Alnawsara H, Tallai B, Gul T, et al. Robotic Assisted Flexible Ureteroscopy in Covid-19 Positive Patient Using Thulium Fiber Laser: Case Report and Literature Review. Am J Clin Case Rep. 2021;1(1):1006.

Copyright: @ 2021 Abdulla Al-Ansari

Publisher Name: Medtext Publications LLC

Manuscript compiled: Dec 20 2021

*Corresponding author: Morshed Ali Salah, Department of Surgery, Hazm Mebaireek General Hospital, Hamad Medical Corporation, Department of Surgery, Qatar University, Qatar, E-mail: morshed.salah@ gmail.com; msalah1@hamad.qa
Coronavirus Disease-19 (Covid-19) was declared to be a pandemic by the WHO on March 11, 2020 [7]. And as a result, many hospitals have been converted to dedicated facilities to manage the Covid-19 patients. The global pandemic has driven many urologists to evaluate the present situation and find a balance between providing optimal and high-quality urological care to their patients, while mitigating the risks of dispersing the infections amongst their other patients and health care workers [8].

Using a robotized and mechanical system to aid in performing FURS in COVID-19 positive patients can minimize the risk of transmitting the disease to the staff. Additionally, the use of the SPFTL can expedite the surgical procedure, there by decreasing the duration of contact with the COVID-19 patient.

The current case report presents a covid-19 positive case who underwent robotic - assisted flexible ureteroscopy using SPTFL for the first time.

\section{Case Presentation}

A 33-year-old male patient presented to the emergency department complaining of right flank pain. The patient had a BMI of $24.8 \mathrm{Kg} / \mathrm{m}^{2}$, his blood investigation was with in the normal range, and his urine culture showed no growth. Non-Contrast Computed Tomography for Kidneys, Ureters, and Bladder (NCCT-KUB) scan 
revealed $15 \mathrm{~mm} \times 12 \mathrm{~mm} \times 8 \mathrm{~mm}\left(754 \mathrm{~mm}^{3}\right)$ and 975 Hounsfield Units (HU) right renal pelvis stone causing mild to moderate back pressure (Figure 1).

Covid-19 Polymerase Chain Reaction (PCR) was positive, but the patient was asymptomatic. Informed consent was obtained from the patient. All staff, who were in direct contact with the patient were in full Personal Protective Equipment (PPE). On induction, 2 gm ceftriaxone intravenously was given. There was a plastic barrier between the patient's head and the anesthesia team.

Under general anesthesia, the patient was put in a lithotomy position, the genital area was disinfected and draped. A $22 \mathrm{Fr}$ cystoscope was inserted into the urinary bladder under vision, the right orifice was identified, a 0.035 inch guidewire was inserted to the right collecting system. A diagnostic ureteroscopy was performed using $7 \mathrm{Fr}$ semi-rigid ureteroscope to check the patency of the ureter and the capability of the ureter to accommodate the Ureteral Access Sheath (UAS). A second, super stiff guidewire was inserted through the ureteroscope to the renal pelvis, a $35 \mathrm{~cm}$ length, 10/12 Fr UAS was inserted over the super stiff guide wire up to the upper ureter. An 8.5 Fr flexible ureteroscope (Flex XC; Karl Storz, Tuttlingen, Germany) was finally introduced through the UAS till the renal pelvis, the stone was identified, then the flexible ureteroscope was fixed in the manipulator unit of the Roboflex (Figure 2).

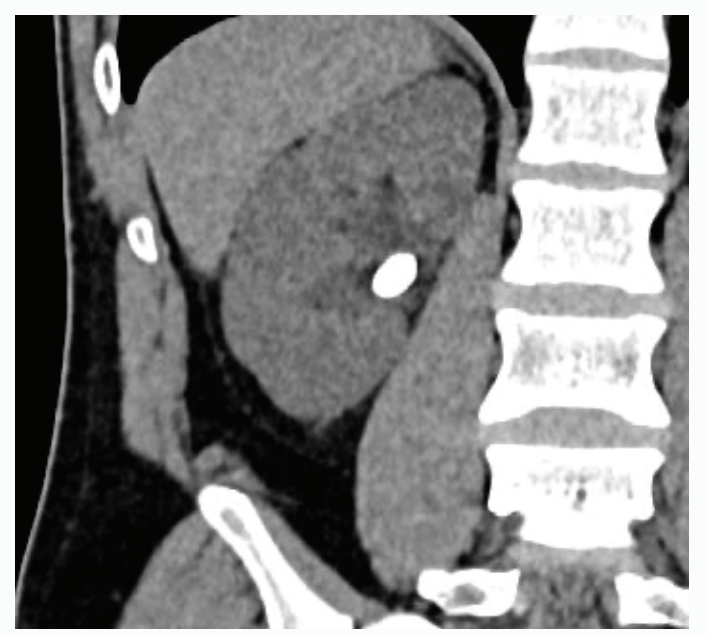

Figure 1: CT-KUB shows $15 \mathrm{~mm} \times 12 \mathrm{~mm} \times 8 \mathrm{~mm}$ obstructing right renal pelvis stone.

A $200 \mu \mathrm{m}$ laser fiber was inserted in the working channel of the flexible ureteroscope. After that, the assistant and the scrub nurse left the area with no direct contact with the patient (social distancing during the surgery) (Figure 3).

Then the stone ablation started remotely with the help of the console and the two pedals. The ergonomically designed chair of Roboflex can be adjusted to a comfortable position, according to the user. Deflection can be performed by manipulating the right handle, similar to a standard FURS. Precise deflection is provided by the thumb wheel on the console. The rotation and forward and backward movement can be controlled by the left handle (Figure 4). The pedals of the laser and fluoroscopy are connected to the console of the Roboflex.

Thulium fiber laser was used (Urolase $\mathrm{SP}^{+}$, IPG) for ablation of

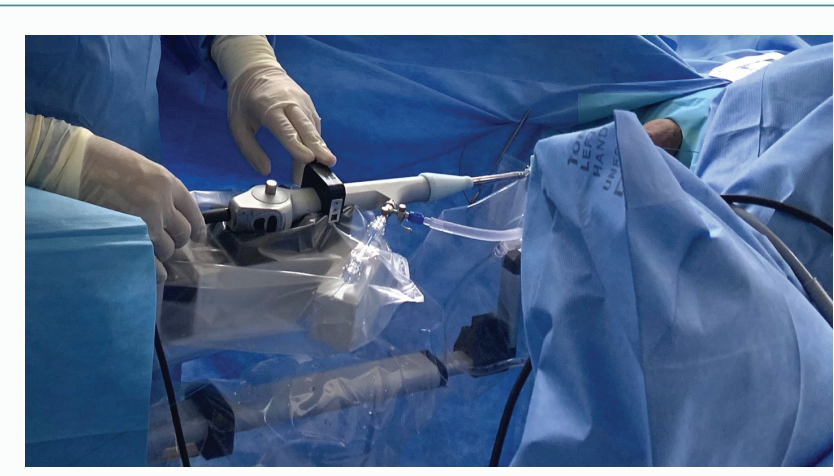

Figure 2: Fixation of the flexible ureteroscope in the manipulator of the avicenna roboflex.

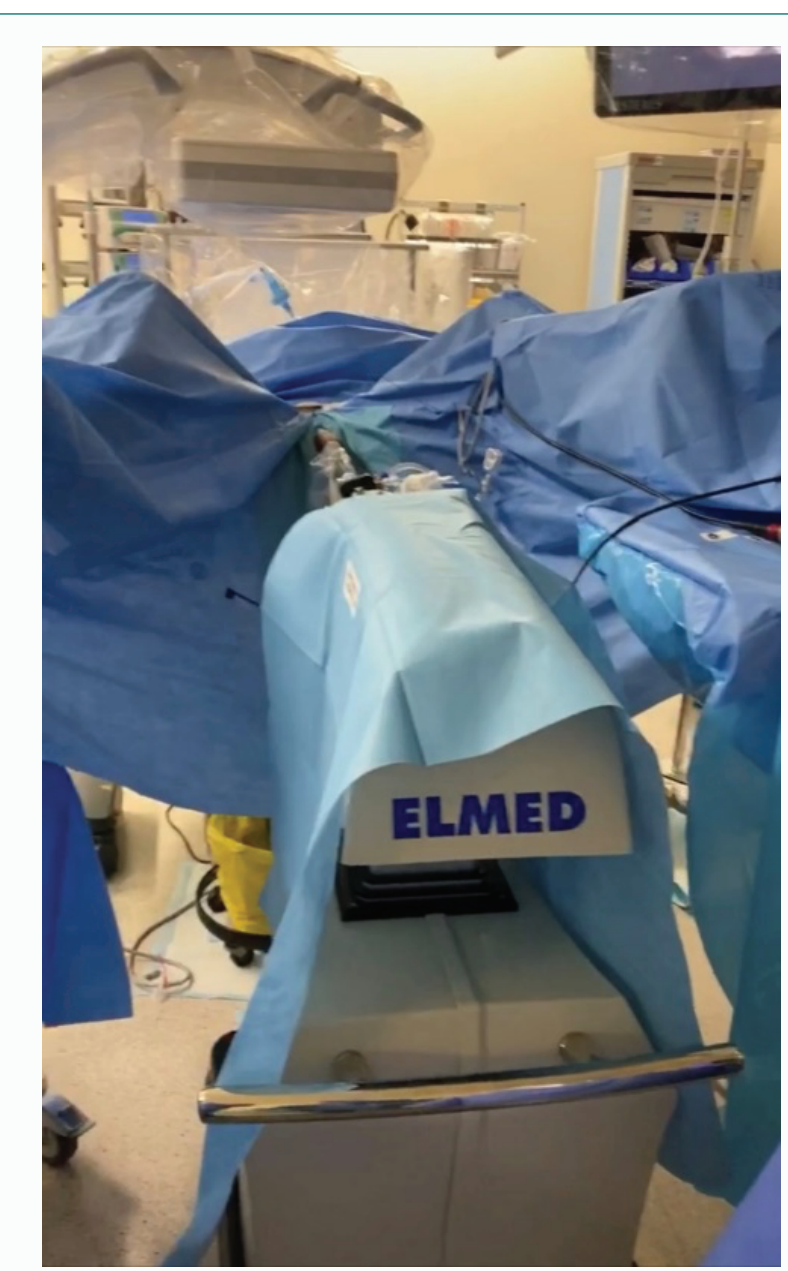

Figure 3: The manipulator after fixing the flexible ureteroscope in lithotomy position, no contact with the patient.

the stone with $0.05 \mathrm{~J}$ and $500 \mathrm{~Hz}$ (25 W pulse energy) for 626 seconds with a total energy of 15670 Joule and 0.1 Joule and $500 \mathrm{~Hz}(50 \mathrm{~W}$ pulse energy) for 69 seconds and total energy of 3460 Joule. The total energy given was 19130 Joule and the lasering time was 695 seconds. At the end, a $6 \mathrm{Fr} / 26 \mathrm{~cm} \mathrm{JJ}$ stent with pull string was inserted. The postoperative period was uneventful, and the patient was discharged on the same day. The JJ stent was removed on the fifth postoperative day. Stone analysis showed $80 \%$ calcium oxalate dihydrate and $20 \%$ calcium oxalate monohydrate. 


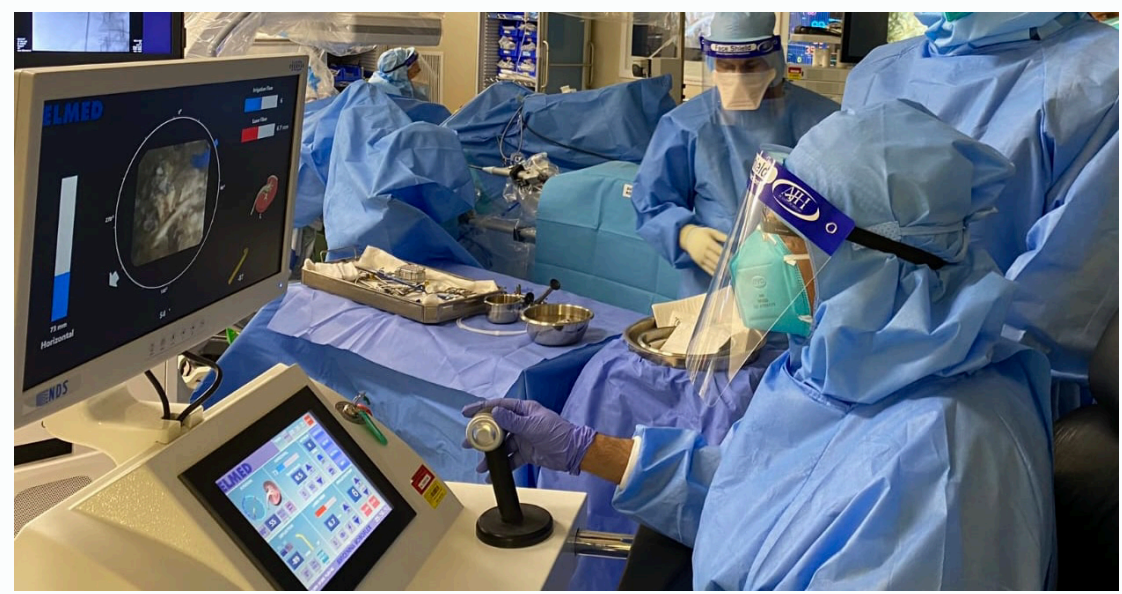

Figure 4: The surgeon is dusting the stone with the help of console of the roboflex.

\section{Discussion}

The standard treatment modalities for a $10 \mathrm{~mm}$ to $20 \mathrm{~mm}$ nonlower pole renal stones, are Extracorporeal Shock Wave Lithotripsy (ESWL), FURS, and Percutaneous Nephrolithotomy (PCNL) [9]. Flexible ureteroscopy is a minimally invasive procedure, with superior outcomes in terms of stone free rates and morbidity, as compared to ESWL and PCNL respectively [10]. Despite the progress that has been made with regards to the design of the flexible ureteroscopes, surgeons still have to work with suboptimal ergonomics and that can impact the surgeon's performance and surgical outcomes $[11,12]$.

One-third of urologists have reported discomfort in their hands and wrist, along with other ergonomic problems while performing the manual version of FURS [11-13]. Surgical robots were mainly introduced to improve the ergonomics of minimally invasive surgeries, which may result in a shorter learning curve of the procedure and might also impact its quality [14-16]. Hence, the use of roboticassisted FURS with Avicenna Roboflex (ELMED, Ankara, Turkey) is favored as it provides significant improvement of ergonomics [3].

Geavlete et al. [4] reported their experience with 298 stones, which were treated with Avicenna Roboflex, and they concluded that robotic-assisted FURS using Avicenna Roboflex has been detected as a safe and efficient treatment method. Additionally, this treatment method allows the surgical team to perform the procedure in a seated position in a comfortable and adjustable chair, whilst maintaining a safe distance from the area exposed to radiation.

Further more, when evaluating the performance rate, the operative results are quite acceptable when compared to the past studies that have measured the performance rate of manual FURS for the treatment of stones [4]. Sarica et al. [17] concluded that Avicenna Roboflex was successful during the combination treatment in a supine position, it aided in holding the FURS in place with fixed tip, which in turn allowed to introduce four targeted calices under endoscopic vision without the help of an assistant. From our experience with around 80 cases underwent Roboflex assisted flexible ureteroscopy, we found that, beside the ergonomic benefits, several other benefits have been found, such as the use of an automatically controlled irrigation system; there is no damage to the flexible ureteroscope with the laser fiber; there is less likelihood of an injury to the mucosa with the laser fiber, and moreover, the length of the procedure was shortened.

When comparing the thulium fiber laser to the holmium laser, the former surpasses the later in many aspects because of the integration of smaller fibers with a core diameter as small as $150 \mu \mathrm{m}$; the pulse energy being as low as $0.025 \mathrm{~J}$; super-high pulse repetition rate ranges up to $2200 \mathrm{~Hz}$ [6]. Martov et al. [18] concluded in their study that TFL technology was associated with an excellent efficacy/safety ratio. Therefore, the SPTFL may be considered as a viable alternative to holmium laser in stone management.

The global pandemic of Covid-19 has led many urologists to make decisions for striking a balance between providing optimal and highquality urological care to their patients while mitigating the risks of dispersing the infection among their patients and health care workers [8]. Even though urinary stone disease represents a benign condition, in a non-negligible number of cases, it can lead to potential severe septic complications that could increase the burden on emergency services [19].

Many surgical specialties including urology have made short pathways for patient flow in order to decrease contact with the patients, thereby lower the probability of transmission of Covid-19. Our hospital was converted to a Covid- 19 facility, therefore all Covid-19 positive patients who were admitted in other hospitals or quarantine facilitates or were under home isolation but needed emergency surgeries had been transferred to our hospital. For major interventions such as orthopedic surgeries, if the patient was in the infectious period (first 10 to 14 days of Covid-19 infection), only the minimal required intervention was carried out. But when the patients became non-infectious the definitive treatment was conducted while ensuring that the patient's safety and the outcomes of the surgery remain uncompromised. For urology conditions, particularly in obstructing stones, only JJ stent was fixed in the infectious period in order to decrease the contact with Covid-19 positive patients. In our reported case, we aimed to use Avicenna Roboflex to minimize the contact with the patient. The use of the TFL aided in expediting the procedure and fragmenting the stones into minute residuals. Additionally, the present case lasering time was 11.58 minutes.

\section{Conclusion}

Using new technologies such as Avicenna Roboflex and thulium 
fiber laser in Covid-19 positive patients during flexible ureteroscopy helps minimize the direct contact with the patient and speed up the procedure, which are important factors that need to be taken into account during Covid-19 Pandemic.

\section{References}

1. Alenezi H, Denstedt JD. Flexible ureteroscopy: technological advancements, current indications and outcomes in the treatment of urolithiasis. Asian J Urol. 2015;2(3):13341.

2. Turk C, Knoll T, Petrik A, Sarica K, Skolarikos A, Straub M, et al. EAU guidelines on urolithiasis. 2013.

3. Saglam R, Muslumanoglu AY, Tokatlı Z, Caskurlu T, Sarica K, Tasci AI, et al. A new robot for flexible ureteroscopy: Development and early clinical results (IDEAL Stage 1-2b). Eur Urol. 2014;66(6):1092-100.

4. Geavlete P, Rassweiler J, Klein J, Tokatli NZ, Traxer O, Al Zarooni A, et al. MP22 18 The volumetric evaluation of multi centric results of robot assisted flexible ureteroscopy. J Urol. 2016;195(4S):e259-60.

5. Rassweiler J, Rassweiler MC, Klein J. New technology in ureteroscopy and percutaneous nephrolithotomy. Curr Opin Urol. 2016;26(1):95-106.

6. Traxer O, Keller EX. Thulium fber laser: the new player for kidney stone treatment? A comparison with Holmium: YAG laser. World J Urol. 2020;38(8):1883-94.

7. World Health Organization. Coronavirus disease (COVID-19) pandemic. Geneva, Switzerland: World Health Organization; April 12, 2020.

8. Teoh JY-C, Ong WLK, Gonzalez-Padilla D, Castellani D, Dubin JM, Esperto F, et al. A global survey on the impact of COVID- 19 on urological services. Eur Urol. 2020;78(2):265-75

9. Turk C, Neisius A, Petrik A, Seitz C, Skolarikos A, Somani B, et al. EAU Guidelines on Urolithiasis. 2021

10. Wright AE, Rukin NJ, Somani BK. Ureteroscopy and stones: Current status and future expectations. World J Nephrol. 2014;3(4):243-8.
11. Elkoushy MA, Andonian S. Prevalence of orthopedic complaints among endourologists are common and their compliance with radiation safety measures. J Endourol. 2011;25(10):1609-13.

12. Healy KA, Pak RW, Cleary RC, Colon-Herdman A, Bagley DH. Hand and wrist problems among endourologists. J Endourol. 2011;25(12):1905-20.

13. Alnadhari I, Ali O, Abdeljaleel O, Sampige VRP, Shamsodini A, Salah M. Ergonomics and surgeon comfort during flexible ureteroscopy. Res Rep Urol. 2021;13:415-24.

14. Rassweiler J, Bach T, Liatsikos E, Rane A, Richstone L, Teber D, Tewari A (2015) Future of minimally invasive surgery. In: Artibani W, Rassweiler J, Kaouk J, Menon M (eds) Minimally invasive surgery in urology, international consultation on minimally invasive surgery in Urology (ICUD-EAU 2015), pp 353-410.

15. Rassweiler JJ, Autorino R, Klein J, Mottrie A, Goezen AS, Stolzenburg JU, et al. Future of robotic surgery in urology. BJU Int. 2017;120(6):822-41.

16. Müller PF, Schlager D, Hein S, Bach C, Miernik A, Schoeb DS. Robotic stone surgery - current state and future prospects: A systematic review. Arab J Urol. 2017;16(3):357-

17. Sarica K, Tokatli NZ, Saglam R, Inal HG, Yilmaz Y, Patel A. MP22-17 Combined robotic flexible ureterorenoscopy and mini percutaneous lithotripsy in supine position. J Urol. 2016;195(4S):e259.

18. Martov, Ergakov DV, Guseynov M, Andrey S. Andronov AS, Plekhanova OA. Clinical comparison of super pulse thulium fiber laser and high-power holmium laser for ureteral stone management. J Endourol. 2021;35(6):795-800.

19. Fukushima H, Kobayashi M, Kawano K, Morimoto S. Performance of quick sequential (sepsis related) and sequential (sepsis related) organ failure assessment to predict mortality in patients with acute pyelonephritis associated with upper urinary tract calculi. J Urol. 2018;199(6):1526-33. 